Hautarzt 2016 - 67:940-947

DOI 10.1007/s00105-016-3894-z

Online publiziert: 9. November 2016

(c) Der/die Autor(en) 2016. Dieser Artikel ist eine Open-Access-Publikation.

\title{
CrossMark
}

\begin{abstract}
Morbus Crohn (MC) und Colitis ulcerosa $(C U)$ sind die beiden wichtigsten Entitäten im Spektrum der chronisch entzündlichen Darmerkrankungen (CED). Sie gelten als Systemkrankheiten und sind nicht selten mit extraintestinalen Manifestationen verbunden. Diese lokalisieren sich vorzugsweise in Gelenken, $v$. a. aber spielen das Hautorgan und hautnahe Schleimhäute eine besonders wichtige Rolle bei diesen Krankheiten. Das Zusammentreffen bzw. das gleichzeitige Auftreten verschiedener Erkrankungen stellt oft eine diagnostische und therapeutische Herausforderung dar.
\end{abstract}

Der MC und die CU stellen die beiden wichtigsten Krankheiten im Spektrum CED dar. Sie treten bei recht ausgeglichener Geschlechtsverteilung zumeist zwischen dem 15. und 40. Lebensjahr erstmals auf [1] und weisen große Ähnlichkeiten in Pathogenese, aber auch in der Klinik auf. Nichtsdestotrotz stellen sie ganz unterschiedliche und eigenständige Entitäten mit charakteristischen klinischen, histologischen, radiologischen etc. Merkmalen dar ( $\bullet$ Tab. 1). Derzeit werden MC und CU als ,autoinflammatorische Krankheiten " betrachtet und daher als Systemerkrankungen verstanden. Vor allem in den „Industriestaaten“ ist es in den letzten Jahrzehnten zu einem kontinuierlichen Anstieg der CEDs gekommen [2]. Die Prävalenzen und Inzidenzen sind ähnlich, jene der CU sind etwas höher: 9 bis 20 Fälle/100.000 Einwohnern/

L. Richter ${ }^{1,2} \cdot$ K. Rappersberger ${ }^{1,2}$

${ }^{1}$ Abteilung für Dermatologie und Venerologie, Krankenanstalt Rudolfstiftung, Wien, Österreich

${ }^{2}$ Abteilung für Dermatologie und Venerologie, Sigmund Freud Privatuniversität, Wien, Österreich

\section{Hauterscheinungen bei chronisch entzündlichen Darmerkrankungen}

\section{Morbus Crohn und Colitis ulcerosa}

Jahr gegenüber 3 bis 15 Fälle/100.000 Einwohnern bei MC. Die Zahl Betroffener ist in Nordamerika und Europa wesentlich höher als in der südlichen Hemisphäre und in östlichen Staaten.

In mehreren Studien konnte gezeigt werden, dass ein hoher Prozentsatz aller Patienten mit CED an einer assoziierten Begleiterkrankung leidet oder diese im Verlauf der Krankheit entwickelt. In großen Studien in der Schweiz (950 Patienten) und Italien (811 Patienten) wurde gezeigt, dass MC-Patienten mit 41-55,3\% etwas häufiger betroffen sind als CU-Patienten mit 31-35,3\% [3, 4]. Praktisch kann jedes Organsystem in den Krankheitsverlauf involviert werden, extraintestinale Manifestationen an der Haut und den hautnahen Schleimhäuten sind besonders häufig und bieten zudem ein ausgesprochen buntes Bild (• Tab. 2; [5, 6]).

Die Manifestationen an der Haut und den hautnahen Schleimhäuten von CEDPatienten werden in 3 Gruppen klassifiziert:

1. Primäre oder auch spezifische Hauterkrankungen (nur bei MC vorkommend)

I. Perianale bzw. peristomale Fisteln und Ulzera

II. Metastatischer Morbus Crohn

III. Orale granulomatöse Veränderungen

2. Sekundär assoziierte entzündliche Hauterkrankungen

I. Erythema nodosum

II. Pyoderma gangraenosum

III. Stomatitis aphthosa
IV. Hidradenitis suppurativa

V. Sweet-Syndrom

VI. Pyostomatitis vegetans

VII. „Bowel-associated dermatosis-arthritis syndrome" (BADAS)

VIII. Blasen bildende Autoimmunerkrankungen

IX. Alopecia areata

X. Vitiligo

XI. Psoriasis

3. Malnutritionssyndrome (oft Kombination mehrerer Defizienzen)

I. Acrodermatitis-enteropathicaähnliche Hautveränderungen im Rahmen eines Zinkmangels

II. Purpura, Hämorrhagien bei Vitamin-K- und/oder Vitamin-C-Mangel

III. Haar- und Nagelveränderungen bei Vitamin-C-, Biotinund/oder Zinkmangel

IV. Stomatitiden und Cheilitis bei Mangel an $\mathrm{B}_{2}, \mathrm{~B}_{6}, \mathrm{~B}_{9}, \mathrm{~B}_{12}$, Vitamin $\mathrm{C}$ und/oder Biotin

\section{Primäre oder auch spezifische Hauterkrankungen}

Spezifische (primäre) Hautläsionen im Rahmen einer CED findet man nur beim MC. Sie sind als Veränderungen definiert, denen weitgehend idente Pathomechanismen wie der Darmentzündung zugrunde liegen, die sich aber extraintestinal manifestieren. Der Schweregrad extraintestinaler Läsionen muss nicht mit der intestinalen Krankheitsaktivität des MC korrelieren, das therapeutische An- 


\begin{tabular}{|c|c|c|}
\hline & Morbus Crohn & Colitis ulcerosa \\
\hline Klinik & $\begin{array}{l}\text { Abdominalschmerzen, Tenesmen, } \\
\text { Diarrhöen (meist nicht blutig!), Erbre- } \\
\text { chen (bei Stenosen im Dünndarm) }\end{array}$ & $\begin{array}{l}\text { Abdominalschmerzen, } \\
\text { Tenesmen, blutig schleimige } \\
\text { Diarrhöen }\end{array}$ \\
\hline Histologie & Transmurales Entzündungsmuster & $\begin{array}{l}\text { Mukosale/submukosale Entzün- } \\
\text { dung }\end{array}$ \\
\hline $\begin{array}{l}\text { Radiologische Zei- } \\
\text { chen }\end{array}$ & 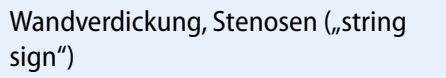 & $\begin{array}{l}\text { Haustrierungsverlust („Fahrrad- } \\
\text { schlauch“), Pseudopolypen }\end{array}$ \\
\hline $\begin{array}{l}\text { Assoziation mit Rau- } \\
\text { chen }\end{array}$ & Ja & Nein \\
\hline Intestinaler Befall & $\begin{array}{l}\text { Ubiquitär, oft auch terminales lleum } \\
\text { ca. } 90 \% \text {, Rektum meist frei }\end{array}$ & Kolon, fast immer auch Rektum \\
\hline $\begin{array}{l}\text { Dominante Immun- } \\
\text { antwort }\end{array}$ & TH1 & $\mathrm{TH} 2$ \\
\hline
\end{tabular}

Tab. 2 Beschriebene extraintestinale Manifestationen der chronisch entzündlichen Darmerkrankungen

\section{Extraintestinale Manifestationen}

Gelenke (ca. $30 \%$ ) Primäre Arthritis, ankylosierende Spondylitis, seropositive rheumatoide Arthritis

Haut- und Schleimhäute $(10-15 \%)$

Augen (ca. 3-6\%)

Leber, Pankreas

(ca. 1-1,5\%)

Gefäße (<1\%)
Erythema nodosum, Pyoderma gangraenosum, Stomatitis aphthosa, Vitiligo, Alopecia areata, Pyostomatitis vegetans etc.

Iritis/Uveitis

Primär biliäre Zirrhose, primär sklerosierende Cholangitis, Pankreatitis

Panarteriitis nodosa, Purpura Schönlein-Henoch, Wegener-Granulomatose, Takayasu-Arteriitis etc.

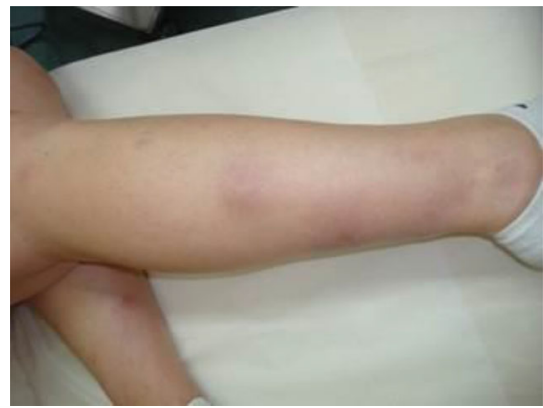

Abb. $1 \Delta$ Erythema nodosum bei einer Patientin mit Morbus Crohn und Psoriasis. Multiple schmerzhafte, entzündliche Knoten an den unteren Extremitäten

tens sind Extremitäten oder intertriginöse Areale betroffen, seltener die $\mathrm{Ge}$ nitalregion oder das Gesicht. Insgesamt handelt es sich um ein äußerst rares Geschehen. Da die Diagnose nur histologisch verifiziert werden kann, sollten verdächtige Hautläsionen beim $\mathrm{MC}$ immer biopsiert werden [9].

\section{Assoziierte entzündliche Hauterkrankungen}

Hierbei handelt es sich um bekannte entzündliche Hautkrankheiten, die häufig bei CED-Patienten auftreten und in 2 Gruppen klassifiziert werden:

- direkt assoziierte reaktive Dermatosen und

- indirekt assoziierte Dermatosen.

\section{Perianale bzw. peristomale Fissuren, Fisteln und Ulzera}

Die häufigsten spezifischen Komplikationen sind perianale bzw. peristomale Fissuren, Fisteln und Ulzera. Diese Läsionen sind klinisch oft recht unspezifisch, eine bereits bekannte Darmerkrankung ist allerdings wegweisend. Eine eindeutige Diagnose kann nur histologisch gestellt werden, da die kutane/subkutane Entzündung durch die Granulome des MC charakterisiert ist. Diese erfassen papilläre und retikuläre Dermis und meist auch die Subkutis entsprechend der transmuralen Entzündung des MC im Darm und führen im Verlauf zu einer Gewebezerstörung mit den typischen klinischen Manifestationen von Fissuren, Erosionen und Ulzera bzw. Fisteln. Die Prävalenz ist sehr hoch und liegt bei $36-45 \%$, wobei auffallenderweise Patienten mit einem
MC des Kolons häufiger betroffen sind als jene mit Manifestationen im Ileum $[6,7]$.

\section{Oraler Morbus Crohn bzw. granulomatöse orale Läsionen}

Die granulomatösen oralen Läsionen werden als Fortsetzung der granulomatösen Darmerkrankung in die oropharyngeale Schleimhaut betrachtet. Sie betreffen etwa 8-9\% aller Patienten und sind klinisch durch unspezifische Erytheme, Schwellungen, kopfsteinpflasterartiges Muster der Schleimhaut, aber auch unspezifische Ulzerationen charakterisiert. Diese Veränderungen können als Initialsymptome eines MC auftreten und haben daher eine besondere diagnostische Bedeutung [8].

Dies sind granulomatöse Entzündungen der Haut, die sich als Knoten, Plaques oder Ulzerationen präsentieren. Meis-

\section{Metastatischer Crohn}

Die direkt assoziierten Dermatosen korrelieren zeitlich und hinsichtlich Intensität mit der Aktivität der Darmentzündung, während die indirekt assoziierten Dermatosen völlig unabhängig von der Darmentzündung verlaufen: Sie sind im Wesentlichen durch ihr gehäuftes Auftreten bei CED-Patienten im Vergleich zur gesunden Bevölkerung definiert [6].

\section{Direkt assoziierte reaktive Dermatosen}

\section{Erythema nodosum}

Etwa 3-8\% aller CED-Patienten entwickeln ein Erythema nodosum (EN; - Abb. 1). Dieses imponiert klinisch durch das plötzliche Auftreten meist multipler, schmerzhafter, entzündlicher Knoten vorzugsweise an den Streckseiten der Unterschenkel, deren histologisches 
Hautarzt 2016·67:940-947 DOI 10.1007/s00105-016-3894-z

(c) Der/die Autor(en) 2016. Dieser Artikel ist eine Open-Access-Publikation.

\section{Richter $\cdot$ K. Rappersberger}

\section{Hauterscheinungen bei chronisch entzündlichen Darmerkrankungen. Morbus Crohn und Colitis ulcerosa}

\section{Zusammenfassung}

Hintergrund. Die Inzidenz und Prävalenz von chronisch entzündlichen Darmerkrankungen (CED) steigen seit mehreren Jahrzehnten in den Industriestaaten kontinuierlich an; beide Krankheiten können zu extraintestinalen Manifestationen führen.

Fragestellung. Wie häufig treten Manifestationen an der Haut und den hautnahen Schleimhäuten beim Morbus Crohn (MC) und der Colitis ulcerosa (CU) auf, und sind diese spezifisch?

Material und Methode. Es erfolgten eine ausführliche Literaturrecherche in PeerReviewed-Journalen via PubMed und eine Zusammenfassung dieser Studien, eine Analyse der externen Daten sowie der Patienten unserer Abteilung.

Ergebnisse. Patienten mit CED entwickeln

häufig mukokutane Entzündungen, doch nur beim MC können primäre Hautmanifestationen, nämlich perianale bzw. peristomale Fisteln und Ulzera, metastatischer Morbus Crohn und orale granulomatöse Veränderungen auftreten. Daneben können verschiedene entzündliche Hauterkrankungen (sekundär) in Assoziation mit MC und CU beobachtet werden. Dazu zählen: Erythema nodosum, Pyoderma gangraenosum, chronische orale Aphthen, Hidradenitis suppurativa, SweetSyndrom, Pyostomatitis vegetans, Bowelassoziiertes Dermatosis-Arthritis-Syndrom u. a. m. Selten werden Malnutritionssyndrome beobachtet (Vitaminmangel, Zinkmangel etc.), diese sind durch typische Klinik und Histopathologie charakterisiert.

Schlussfolgerungen. Verschiedene entzündliche Krankheiten an der Haut und den hautnahen Schleimhäuten können mit einem MC oder einer CU assoziiert sein. Allerdings sind die wissenschaftlichen Daten über den unmittelbaren pathogenetischen Zusammenhang und die Häufigkeit der verschiedenen mukokutanen Manifestationen sehr heterogen und teilweise auch widersprüchlich. Dennoch sind gute Kenntnisse um Krankheitsassoziationen und deren Klinik wie auch von Mangelerscheinungen Voraussetzungen für eine rasche Diagnose und frühzeitige Therapie sowie die Verhinderung von Folgeschäden.

\section{Schlüsselwörter}

Pyoderma gangraenosum - Sweet-Syndrom . Erythema nodosum . Stomatitis aphthosa . Hidradenitis suppurativa

\section{Cutaneous involvement in chronic inflammatory bowel disease. Crohn's disease and ulcerative colitis}

\section{Abstract}

Background. Over recent decades, both the incidence and prevalence of chronic inflammatory bowel disease have continued to rise in industrialized countries; the disease is frequently associated with extracutaneous involvement and comorbidity.

Objectives. The purpose of this work was to investigate the frequency and specificity of mucocutaneous manifestations in Crohn's disease (CD) and ulcerative colitis (UC).

Materials and methods. An extensive search in peer-reviewed journals via PubMed was performed; presented is a summary and analysis of various studies and data, including data of patients treated at our department. Results. CD and UC are frequently associated with mucocutaneous symptoms; however, primary/specific disease-associations are exclusively seen in CD patients. These include peri-anal and -stomal fistulas and ulcerations, "metastatic" Crohn's disease as well as oral granulomatous disease. Moreover, in both CD and UC, there occur several other inflammatory skin conditions such as erythema nodosum, pyoderma gangrenosum, hidradenitis suppurativa, chronic oral aphthous disease, Sweet syndrome, pyostomatitis vegetans, and bowel-associated dermatosis-arthritis syndrome. Malnutrition syndromes (zinc and vitamin deficiencies) are only rarely observed. Conclusion. On skin and oral/genital mucous membranes various different inflammatory manifestations may be observed during the course of CD or UC. However, most data about a direct pathogenic relationship of the gastrointestinal and dermatologic disorders are quite heterogeneous or even contradictory. Nevertheless, knowledge of these conditions and their possible association with CD and UC could be crucial for early diagnosis and initiation of an appropriate therapy and thus be essential to prevent secondary tissue damage.

\section{Keywords}

Pyoderma gangrenosum - Sweet syndrome . Erythema nodosum · Stomatitis aphthosa . Hidradentitis suppurativa
Substrat eine septale Pannikulitis ohne Vaskulitis darstellt $[5,6]$. Die derzeit noch gängige Klassifikation des $\mathrm{EN}$ als direkt assoziierte reaktive Dermatose wird zunehmend infrage gestellt, da zuletzt in großen Studien darauf hingewiesen wurde, dass viele EN-Episoden keineswegs in einem direkten zeitlichen Zusammenhang mit Aktivitätsschüben einer CED stehen $[3,10]$. Das EN tritt beim MC signifikant häufiger $(5,6 \%)$ als bei der CU (1,2 \%) auf. Besonders auffallend ist die Geschlechterverteilung, die zeigt, dass 82,5\% der Patienten mit CED und EN weiblich sind [10]. Die Hälfte aller Betroffenen entwickelt gleichzeitig eine Arthritis [5, 10]. Die Therapie erfolgt mit systemischen Glukokortikoiden (1 mg/kg Körpergewicht [KG] Initialdosis) in typischer, „ausschleichender“ Form [11].

\section{Pyoderma gangraenosum}

Das Pyoderma gangraenosum (PG; - Abb. 2) stellt meist eine schwere kutane, sehr selten auch mukokutane (s. unten) Komplikation einer CED dar. In einer rezenten europäischen Multicenterstudie über einen Beobachtungszeitraum von 10 Jahren konnte bei 1145 Patienten mit CED kein Unterschied der Prävalenz eines PG beim MC oder bei der CU nachgewiesen werden [12]. 

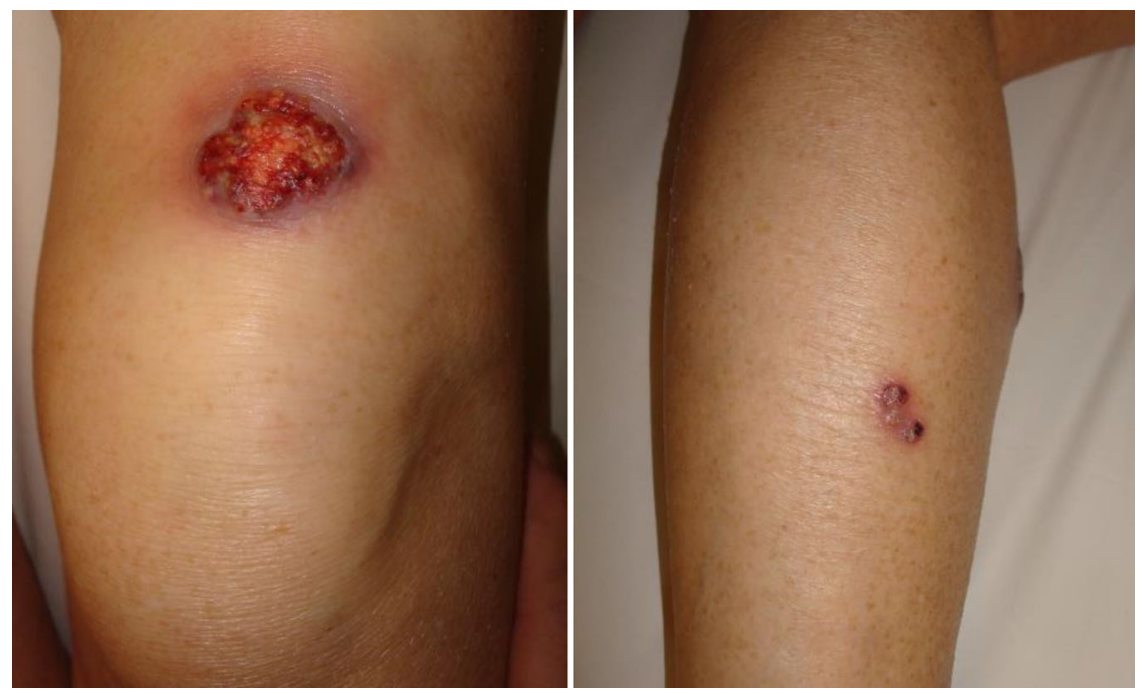

Abb. 2 ム Multilokuläres Pyoderma gangraenosum: tiefe, rasch progrediente Ulzera mit einem matschigen Wundgrund, unterminierten Rändern und einem entzündlichen Randsaum

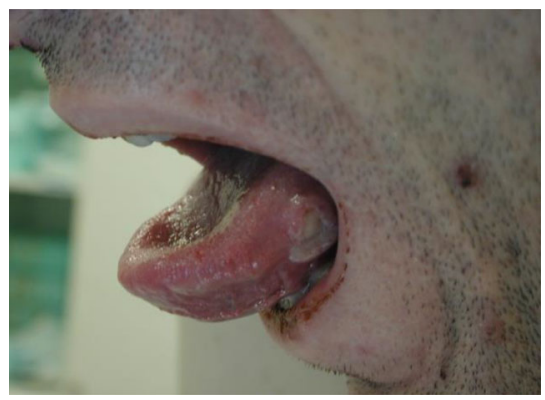

Abb. $4 \Delta$ Pyostomatitis vegetans, charakterisiert durch schmerzhaft, aphthöse Ulzera an der Zungenschleimhaut

Die äußerst schmerzhaften Läsionen können solitär, aber auch multilokulär auftreten und stellen oft eine große diagnostische Herausforderung für die behandelnden Internisten, v. a. aber für Chirurgen dar. Daher werden frühe PG, die initial häufig als hämorrhagische Knoten oder hämorrhagische Pusteln imponieren, immer wieder als infektiöse Ereignisse interpretiert und inzidiert, was bei zugrunde liegendem Pathergiephänomen gelegentlich fatale Auswirkungen hat und $\mathrm{zu}$ riesigen Wundflächen führt. Eine chirurgische Intervention ist obsolet, die Therapie erfolgt pharmakologisch und setzt sich aus hoch dosierten Steroidgaben (anfänglich einer Pulstherpie mit 1-2 g/Tag i. v.), evtl. in Kombination mit einem konventionellen Immunsuppressivum wie Cyclosporin A, Mycophenolat-Mofetil oder Azathioprin, zusammen. Auch
TNF-a-Blocker scheinen - insbesondere bei therapierefraktären Fällen - eine gute Behandlungsalternative darzustellen [13].

\section{\) Die Therapie erfolgt pharmakologisch}

Die Behandlung dauert meist Wochen bis Monate, die Abheilung erfolgt mit typischen "gestrickten“ Narben, sie ist gelegentlich mit Funktionseinschränkungen verbunden.

\section{Stomatitis aphthosa}

Die Stomatitis aphthosa ist durch das plötzliche Auftreten meist multipler, scharf begrenzter schmerzhafter Ulzera, die von einem höchst entzündlich roten Randsaum umgeben sind, definiert. Bei CED werden meist chronisch rezidivierende Aphthen (CRA) mit einem Durchmesser $<1 \mathrm{~cm}$ beschrieben. In verschiedenen Übersichtsarbeiten wird ein Auftreten der CRA bei etwa $10 \%$ der CED-Patienten angegeben. Damit ist diese Inzidenz kaum höher als in der Normalbevölkerung und wurde auch kürzlich erst für Schweizer Patienten bestätigt $[3,5,8,14,15]$. Deshalb möchten wir anregen, dass die derzeitige Klassifizierung einer CRA als assoziierte Krankheit der CED in unseren Breiten kritisch hinterfragt werden sollte [5, 14]. Auffallend sind dagegen die Da-

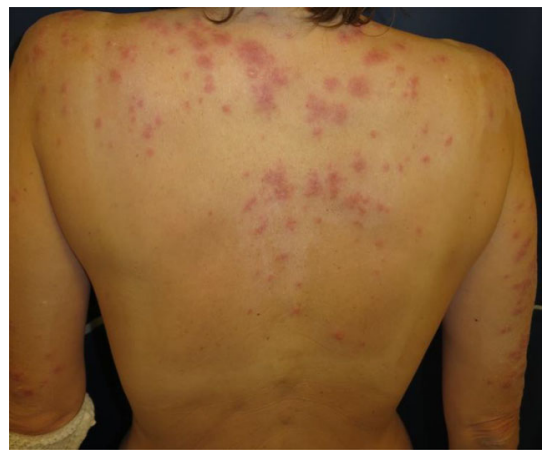

Abb. 3 ॥ Sweet-Syndrom im Rahmen einer Colitis ulcerosa: multiple hoch entzündlich gerötete Papeln und Knoten am Rücken

ten in der Türkei, wo CRA bei 35,86\% der CED-Patienten beschrieben wurden [15]. Ebenso zeigt sich bei Kindern und Jugendlichen eine eindeutige Korrelation zwischen CED und CRA, wobei die CRA der Darmerkrankung oft vorangeht und erster Hinweis für eine CED sein kann $[8,16]$. Solche Patienten sollten daher recht engmaschig ärztlich kontrolliert werden [15].

Über die Pathogenese der CRA ist wenig bekannt. Als Auslöser dieses lokalen Entzündungsgeschehens wurden verschiedene Ursachen vermutet, v. a. eine genetische Disposition und physikalisch chemische Traumata der Mundschleimhaut, Störungen im Eisenstoffwechsel, aber auch Folsäure- und Vitamin-B ${ }_{12}$-Mangel [17]. Eine kausale Behandlung gibt es nicht, Spülungen mit (Salbei-)Tee oder Kochsalzlösungen, topische Anästhetika, Kortikoide und Calcineurininhibitoren können Linderung verschaffen.

\section{Hidradenitis suppurativa}

Die Hidradenitis suppurativa (HS) tritt mit einer Prävalenz von $1-2 \%$ in der Gesamtbevölkerung auf, Frauen sind etwas häufiger betroffen als Männer (3:1). Die HS ist durch eine chronisch rezidivierende, sehr schmerzhafte Entzündung des Haarfollikel-/Talgdrüsenapparates in Assoziation mit apokrinen Drüsen charakterisiert. Sie betrifft daher genau definierte Lokalisationen: axillär, submammär und inguino-genital. Der Schweregrad der Erkrankung bestimmt die weitere Therapie und muss daher immer genau definiert werden. Die Klassifizierung in 3 Grade nach Hurley erscheint uns im kli- 


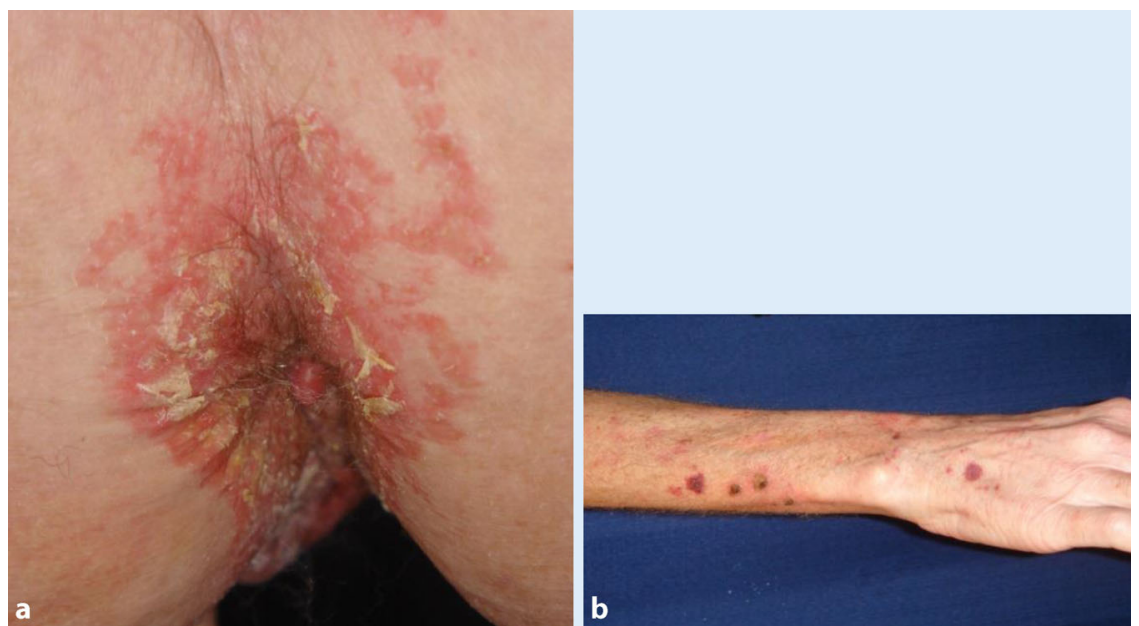

Abb. $5 \Delta$ Kombiniertes Malnutritionssyndrom (Zink- und Vitamin-C-Mangel). a Typische periorifizielle, erosive Dermatitis. b Hämorrhagien bzw. non-palpable Purpura. Therapie durch die Substitution des mangelnden Vitamins oder Spurenelements sehr einfach

nischen Alltag am einfachsten und besten geeignet [18, 19].

\section{》) Der Schweregrad der Erkrankung muss genau definiert werden}

Die Assoziation der HS mit CED ist länger bekannt, die Inzidenz wurde in einer rezenten Analyse mit 12,8\% beschrieben, wobei 17,3\% der Patienten mit MC und $8,5 \%$ der Patienten mit CU betroffen sind. Somit haben CED-Patienten ein 9-fach erhöhtes Risiko gegenüber der Normalbevölkerung, an einer HS zu erkranken [20].

Über die Ätiopathogenese der HS ist wenig bekannt, genetische Disposition scheint eine Rolle zu spielen. Als determinierender Kofaktor für beide Krankheiten - HS und CED - wurde chronischer Nikotinabusus identifiziert [18]. Die Behandlung richtet sich nach dem klinischen Schweregrad (Hurley $\mathrm{I}^{\circ}-\mathrm{III}^{\circ}$ ) der HS. Mildere Formen (Hurley $\mathrm{I}^{\circ}-\mathrm{II}^{\circ}$ ) werden mit topischen Antiseptika und systemischen Antibiotika pharmakologisch behandelt, zusätzlich müssen oft kleinere chirurgische Eingriffe, v. a. Abszessinzisionen durchgeführt werden. Bei Hurley III $^{\circ}$ gelten großzügige chirurgische Exzisionen der betroffenen Areale am besten unter Einhaltung eines Sicherheitsabstandes als Goldstandard, weil damit die Krankheit geheilt werden kann. Die vor Kurzem erfolgte Zulas- sung des TNF- $\alpha$-Antagonisten Humira ${ }^{\circledR}$ bereichert unsere therapeutischen Möglichkeiten, insbesondere weil mit $1 \mathrm{Me}$ dikament 2 verschiedene Krankheiten gleichzeitig behandelt werden können. Noch ist es aber zu früh, um über die Wirksamkeit und Sinnhaftigkeit dieser kostspieligen Therapie bei der HS außerhalb von Studien, also im klinischen Alltag, zu berichten.

\section{Sweet-Syndrom/akute febrile neutrophile Dermatose}

Eine seltenere Erkrankung mit Hautsymptomen, assoziiert mit CED, ist das Sweet-Syndrom/akute febrile neutrophile Dermatose (• Abb. 3 [21]). Klinisch imponieren bis walnussgroße, entzündlich gerötete, oft pseudovesikulöse/-bullöse Knoten, die solitär oder multipel, v. a. disseminiert am Stamm (• Abb. 3), oft begleitet von Fieberschüben, innerhalb weniger Tage auftreten. Im peripheren Blut findet man eine Neutrophilie, in der Histologie ein diffuses Infiltrat aus Neutrophilen und Kernstaub in der gesamten Dermis. In der papillären Dermis ist oft ein mächtiges Ödem zu erkennen. In einer rezenten retrospektiven Studie mit 90 Patienten mit Sweet-Syndrom wurden nur 4 Patienten (3,6\%) mit einer CED identifiziert [22]. Wesentlich häufiger ist die Assoziation der febrilen neutrophilen Dermatose mit einer malignen hämatologischen Krankheit. Sie wird aber auch bei Infektionen meist im Rahmen von respiratorischen Infekten mit Streptokokken, seltener bei gastrointestinalen Infektionen mit Salmonellen, beobachtet $[22,23]$.

\section{Blasen bildende Autoimmuner- krankungen}

In Assoziation mit CED wurden wiederholt Blasen bildende Autoimmunerkrankungen kasuistisch und in kleinen Fallsammlungen beschrieben, allerdings wurde meist die zugrunde liegende Darmkrankheit nicht in MC oder CU spezifiziert. Augenscheinlich scheint aber ein pathophysiologischer Zusammenhang, v. a. des MC mit der Epidermolysis bullosa acquisita, da die pathogenetischen Autoantikörper sowohl mit Typ-VII-Kollagen in der Basalmembran der Epidermis wie auch des Darmepithels reagieren [24, 25]. Dagegen werden lineare IgA-Dermatose, bullöses Pemphigoid und IgA-Pemphigus häufiger bei CU-Patienten beschrieben, die Inzidenz ist allerdings sehr gering [26].

Typischerweise erkranken die Patienten zuerst an einer CED und entwickeln erst im weiteren Verlauf eine Blasen bildende Autoimmunerkrankung. Erklärt wird dieses Phänomen gerne mit Hinweisen aus experimentellen Studien, die zeigten, dass erst nach dem $\mathrm{Zu}$ sammenbruch und der Zerstörung der epithelialen Schichtung der Darmmukosa bei CED entsprechende Antigene frei werden, um eine Immunantwort einzuleiten, die im Hautorgan pathophysiologisch wirksam wird [25]. Zusätzlich unterstützt wird diese Hypothese durch die klinische Beobachtung, dass nach Kolektomien auch die Hautmanifestationen abheilen und ausbleiben.

\section{Weitere häufige dermatologische Erkrankungen}

Mit CED in Verbindung gebracht werden außerdem Alopecia areata, Vitiligo und Psoriasis. Eine rezente polnische Studie zeigt, dass Patienten mit CU oder MC ein bis zu 3-fach erhöhtes Risiko haben, an einer Alopecia areata zu erkranken, als die Normalbevölkerung [27]. Auch bei der Vitiligo scheinen CU- und MC-Patienten gleich häufig eine Vitiligo zu entwickeln, das Risiko dafür ist etwa doppelt so hoch wie in der Normalbevölkerung [28]. 
Wissenschaftliche Daten zur Assoziation einer Psoriasis mit einer CED sind bescheiden: Es gibt recht eindeutige Daten hinsichtlich einer Assoziation einer CED bei Patienten mit Psoriasis. So zeigen die Nurses' Health Study (NHS) I (1996-2008) und NHS II (1991-2007) ein 4-fach erhöhtes Risiko für Psoriatiker, an einem MC zu erkranken, aber kein erhöhtes Risiko für eine CU [29]. Umgekehrt ist die Datenlage ausgesprochen widersprüchlich und lässt derzeit keine eindeutige Aussage zu [30, 31].

Die Pyostomatitis vegetans und das „bowel-associated dermatosis-arthritis syndrome“ (BADAS) treten sehr häufig bei CED auf: Die Pyostomatitis vegetans (- Abb.4), erstmals 1949 beschrieben, ist bei Männern doppelt so häufig wie bei Frauen und etwas häufiger bei der CU als beim MC $[32,33]$. Klinisch imponieren meist multiple Pusteln mit erythematösem, verdicktem Randsaum vornehmlich an der labialen und bukkalen Schleimhaut, wogegen der Zungenrücken meist ausgespart bleibt. Sie rupturieren rasch und entwickeln sich zu Erosionen und Ulzera. Die Läsionen sind gelegentlich in (Schlangen-)Linien angeordnet, nach längerem Verlauf kann ein kopfsteinpflasterartiges Bild entstehen. Selten ist die Anogenitalregion betroffen [33].

Die BADAS ist eine schubweise auftretende „neutrophile Dermatose“, initial charakterisiert durch Erytheme, aus denen sich rasch Vesikel und Pusteln entwickeln. Prädilektionsstellen sind Extremitäten und der proximale Stammbereich, aber auch die Mundschleimhaut kann betroffen sein. Das Auftreten der Haut und Schleimhautläsionen ist häufig von Fieber und Arthralgien begleitet. BADAS ist auch eine Komplikation der bariatrischen Chirurgie, fast $20 \%$ der Patienten mit einem jejunoilealen Bypass entwickeln dieses Krankheitsbild. Als Pathomechanismus vermutet man, dass es $\mathrm{zu}$ einer extensiven Besiedelung mit Mikroorganismen des „stillgelegten“ Teils des Darms kommt. Dies soll zu einer überschießenden Immunantwort gegen Bakterienantigene (Escherichia coli, Bacteroides fragilis oder Streptococcus sp.) führen. Es wird angenommen, dass die Bildung von Immunkomplexen eine wesentliche Rolle spielt $[6,34]$. Für diese These spre-
Hier steht eine Anzeige. Springer 
Tab. 3 Dermatologische Symptome bei verminderter Aufnahme von Vitaminen oder Spurenelementen

\begin{tabular}{|c|c|c|}
\hline $\begin{array}{l}\text { Vitamin } \\
\text { oder Spuren- } \\
\text { element }\end{array}$ & Haut & Schleimhaut \\
\hline Vitamin A & $\begin{array}{l}\text { Streckseitige follikuläre, spinuläre oder flach keratoti- } \\
\text { sche, hautfarbene oder pigmentierte Papeln }\end{array}$ & 0 \\
\hline Vitamin $\mathrm{K}$ & Hämorrhagien, Purpura, Ekchymosen & Schleimhautblutungen \\
\hline $\begin{array}{l}\text { Vitamin } B_{2} \\
\text { (Riboflavin) }\end{array}$ & $\begin{array}{l}\text { Perlèche, Cheilitis, seborrhoische Dermatitis-artige } \\
\text { Läsionen }\end{array}$ & Glossitis \\
\hline $\begin{array}{l}\text { Vitamin } B_{3} \\
\text { (Niacin) }\end{array}$ & $\begin{array}{l}\text { Pellagra } \\
\text { Akut: symmetrisch rötlich bräunliche Makulae bzw. } \\
\text { Plaques v. a. an sonnenexponierten Arealen. Selten } \\
\text { Blasen und Ulzerationen (Casal-Halsband!) } \\
\text { Chronisch: Verdickung der Haut, Rhagaden, Fissuren, } \\
\text { Palmarkeratosen, periorifizielle Dermatitis, Cheilitis }\end{array}$ & Glossitis, Mukositis \\
\hline Vitamin $B_{6}$ & Pellagra-ähnlich & $\begin{array}{l}\text { Glossitis, Stomatitis, } \\
\text { kleine Ulzerationen }\end{array}$ \\
\hline $\begin{array}{l}\text { Vitamin } B_{9} \\
\text { und } B_{12}\end{array}$ & $\begin{array}{l}\text { Perlèche, Depigmentation der Haare, Hyperpigmenta- } \\
\text { tion (Hände, Nägel, Gesicht, Flexuren, Druckpunkte) }\end{array}$ & $\begin{array}{l}\text { Hyperpigmentation } \\
\text { der Mukosa } \\
\text { Hunter-Glossitis: } \\
\text { hochrote, spiegelglat- } \\
\text { te Zungenoberfläche, } \\
\text { Verlust der Papillen, } \\
\text { Zungenbrennen }\end{array}$ \\
\hline Vitamin C & $\begin{array}{l}\text { Skorbut } \\
\text { Vergrößerte, hyperkeratotische Haarfollikel strecksei- } \\
\text { tig, dann generalisiert; brüchiges Haar; Hämorrhagien, } \\
\text { Purpura, Splitterblutungen der Nägel }\end{array}$ & Gingiva-Hämorrhagien \\
\hline Biotin & Periorifizielle Dermatitis, Alopezie & Glossitis \\
\hline Zink & $\begin{array}{l}\text { Dünnes, brüchiges Haar, periorifizielle Dermatitis, } \\
\text { symmetrische ekzematöse Plaques, die oft bullös und } \\
\text { dann erosiv werden, psoriasiform bei mildem Verlauf }\end{array}$ & 0 \\
\hline
\end{tabular}

chen auch die histologischen Hinweise auf eine Vaskulitis [35]. Unterstützt wird diese Hypothese auch durch das gute klinische Ansprechen der Patienten auf eine Langzeitantibiose einerseits und das Abklingen der Hautveränderungen nach chirurgischer Revision mit Beseitigung der „blind loop“.

\section{Malnutritionssyndrome (oft Kombination mehrerer Defizienzen)}

In den Industriestaaten sind Malnutritionssyndrome eine Seltenheit geworden. Die damit verbundenen dermatologischen Symptome stellen daher meist eine Herausforderung für den Kliniker dar. Die Hauptursachen für Malnutritionssyndrome in den Industrieländern sind chronischer Alkoholismus und psychiatrische Erkrankungen (v. a. Essstörungen, aber auch Depressionen u. a.m.). Selten führt eine CED per se durch verminderte Aufnahme von Vitaminen und/oder Spurenelementen zu einem Malnutritionssyndrom, allerdings wird dieses in Folge chirurgischer Therapiemaßnahmen beim MC oder der CU gelegentlich beobachtet. Wesentlich häufiger werden Malnutritionssyndrome nach bariatrischer Chirurgie, insbesondere bei schwangeren Frauen und stillenden Müttern, beobachtet [36, 37].

In $\bullet$ Tab. 3 werden die häufigsten Mangelsyndrome, die im Rahmen von CEDs beschrieben wurden, zusammengefasst (• Abb. 5).

\section{Fazit für die Praxis}

\section{- Nur MC verursacht spezifische} Hauterscheinungen: perianale bzw. peristomale Fisteln und Ulzera, metastatischer Crohn, orale granulomatöse Veränderungen.

- Die häufigsten mit CED assoziierten Dermatosen sind: Erythema nodosum, Stomatitis aphthosa, Hidradenitis suppurativa.

- Das Pyoderma gangraenosum stellt eine der schwersten extraintestinalen Komplikationen im Rahmen einer CED dar. Eine rasche immunsuppressive Therapie ist mandatorisch.

\section{- BADAS und Pyostomatitis vegetans} sind seltene Haut-SchleimhautManifestationen, die fast immer auf eine CED hinweisen.

- Malnutritionssyndrome entwickeln sich so gut wie ausschließlich nach chirurgischer Sanierung einer CED.

- Malnutritionssyndrome im Rahmen einer CED sind selten; dennoch sollten sie rasch erkannt werden, da eine therapeutische Substitution sehr einfach ist.

\section{Korrespondenzadresse}

\section{Dr. L. Richter}

Abteilung für Dermatologie und Venerologie, Krankenanstalt Rudolfstiftung Juchgasse 25, 1030 Wien, Österreich leo.richter@wienkav.at

\section{Einhaltung ethischer Richtlinien}

Interessenkonflikt. L. Richter und K. Rappersberger geben an, dass kein Interessenkonflikt besteht.

Dieser Beitrag beinhaltet keine von den Autoren durchgeführten Studien an Menschen oder Tieren.

Open Access. Dieser Artikel wird unter der Creative Commons Namensnennung 4.0 International Lizenz (http://creativecommons.org/licenses/by/4.0/deed. de) veröffentlicht, welche die Nutzung, Vervielfältigung, Bearbeitung, Verbreitung und Wiedergabe in jeglichem Medium und Format erlaubt, sofern Sie den/die ursprünglichen Autor(en) und die Quelle ordnungsgemäßnennen, einen Linkzur Creative Commons Lizenz beifügen und angeben, ob Änderungen vorgenommen wurden.

\section{Literatur}

1. BaumgartDC (2012) Crohn's disease and ulcerative colitis - from epidemiology and Immunobiology to a rational diagnostic and therapeutic approach. Springer, New York

2. Ordás I, Eckmann L, Talamini M, Baumgart DC, Sandborn WJ (2012) Ulcerative colitis. Lancet 380:1606-1619

3. Vavricka SR, Brun L, Ballabeni P, Pittet V, Prinz Vavricka BM, Zeitz J, Rogler G, Schoepfer AM (2011) Frequency and risk factors for extraintestinal manifestations in the swiss inflammatory bowel disease cohort. Am JGastroenterol 106(1):110-119 
4. Zippi M, Corrado C, Pica R, Avallone EV, Cassieri C, De Nitto D, Paoluzi P, Vernia P (2014) Extraintestinal manifestations in a large series of Italian inflammatory bowel disease patients. World J Gastroenterol 20(46):17463-17467

5. Danese $S$, Semeraro S, Papa A, Roberto I, Scaldaferri F, Fedeli G, Gasbarrini G, Gasbarrini A (2005) Extraintestinal manifestations in inflammatory bowel disease. World J Gastroenterol 11(46):7227-7236

6. Thrash B, Patel M, Shah KR, Boland CR, Menter A (2013) Cutaneous manifestations of gastrointestinal disease: Part II. J Am Acad Dermatol 68(2):211.e1-211.33 (quiz 244-6)

7. Tavarela Veloso F (2004) Review article: skin complications associated with inflammatory bowel disease. Aliment Pharmacol Ther 20(Suppl 4): $50-53$

8. Muhvić-Urek M, Tomac-Stojmenović M, Mijandrušić-Sinčić B (2016) Oral pathology in inflammatory bowel disease. World J Gastroenterol 22(25):5655-5667

9. Lang N, Hartschuh W, Enk A, Toberer F (2015) Metastatic Crohn's disease: a diagnostic and therapeutic challenge. J Dtsch Dermatol Ges 13(6):571-574

10. Farhi D, Cosnes J, Zizi N, Chosidow O, Seksik P, Beaugerie L, Aractingi S, Khosrotehrani K (2008) Significance of erythema nodosum and pyoderma gangrenosum in inflammatory bowel diseases. Medicine (Baltimore) 87(5):281-293

11. Veloso FT (2011) Extraintestinal manifestations of inflammatory bowel disease: Do they influence treatment and outcome? World J Gastroenterol 17(22):2702-2707

12. Isene $R$, Bernklev $T$, Høie O, Munkholm $P$, Tsianos $E$, Stockbrügger R, Odes S, Palm $\emptyset$, Småstuen $M$, Moum B, EC-IBDStudy Group (2015) Extraintestinal manifestations in Crohn's disease and ulcerative colitis: results from a prospective, populationbased European inception cohort. Scand $J$ Gastroenterol 50(3):300-305

13. Patel F, Fitzmaurice $S$, Duong $C$, He $Y$, Fergus J, Raychaudhuri SP, Garcia MS, Maverakis E (2015) Effective strategies for the management of pyoderma gangrenosum: a comprehensive review. Acta Derm Venereol 95(5):525-531

14. Fritsch $P$ (2009) Dermatologie und Venerologie für das Studium. Springer, Berlin, S746

15. Topaloğlu Demir F, KocatürkE, Yorulmaz E, Adalı G, Kavala M (2014) Mucocutaneous manifestations of inflammatory bowel disease in turkey. J Cutan Med Surg 18(6):397-404

16. Gürkan $A$, Özlü SG, Altıaylık-Özer P, Kurtul BE, Karacan CD, Şenel S (2015) Recurrent aphthous stomatitis in childhood and adolescence: A single-center experience. Pediatr Dermatol 32(4):476-480

17. Slebioda Z, Szponar E, Kowalska A (2014) Etiopathogenesis of recurrent aphthous stomatitis and the role of immunologic aspects: literature review. Arch Immunol Ther Exp (Warsz) 62(3):205-215

18. Jemec GB, Kimball AB (2015) Hidradenitis suppurativa: Epidemiology and scope of the problem. J Am Acad Dermatol 73(5):4-7

19. Principi M, Cassano N, Contaldo A, lannone A, Losurdo G, Barone M, Mastrolonardo M, Vena GA, lerardi E, Di Leo A (2016) Hydradenitis suppurativa and inflammatory bowel disease: An unusual, but existing association. World J Gastroenterol 22(20):4802-4811

20. Kohorst JJ, Kimball AB, Davis MD (2015) Systemic associations of hidradenitis suppurativa.J Am Acad Dermatol 73(5):S27-S35
21. Ytting H, Vind I, Bang D, Munkholm P (2005) Sweet's Syndrome - An extraintestinal manifestation in inflammatory bowel disease. Digestion 72(2-3):195-200

22. Amouri M, Masmoudi A, Ammar M, Boudaya $S$, Khabir A, Boudawara T, Turki H (2016) Sweet's syndrome: a retrospective study of 90 cases from a tertiary care center. Int J Dermatol 55(9):1033-1039

23. Cohen PR (2007) Sweet's syndrome - a comprehensive review of an acute febrile neutrophilic dermatosis. Orphanet J Rare Dis 26(2):34

24. Hundorfean G, Neurath MF, Sitaru C (2010) Autoimmunity against type VII collagen in inflammatory bowel disease. J Cell Mol Med 14(10):2393-2403

25. Chen M, O'Toole EA, Sanghavi J, Mahmud N, Kelleher D, Weir D, Fairley JA, Woodley DT (2002) The epidermolysis bullosa acquisita antigen (type VIIcollagen) is present in human colon and patients with crohn's disease have autoantibodies to type VII collagen. J Invest Dermatol 118(6):1059-1064

26. Shipman AR, Reddy H, Wojnarowska F (2012) Association between the subepidermal autoimmune blistering diseases linear IgA disease and the pemphigoid group and inflammatory bowel disease: two case reports and literature review. Clin Exp Dermatol 37(5):461-468

27. Sobolewska-Włodarczyk A, Włodarczyk M, Fichna J, Wiśniewska-Jarosińska M (2016) Alopecia areata in patients with inflammatory bowel disease: an overview. Folia Med Cracov 56(1):5-12

28. Gill L, Zarbo A, Isedeh P, Jacobsen G, Lim HW, Hamzavi I (2016) Comorbid autoimmune diseases in patients with vitiligo: a cross-sectional study. J Am Acad Dermatol 74(2):295-302

29. LiWQ, Han JL, Chan AT, Qureshi AA (2013) Psoriasis, psoriatic arthritis and increased risk of incident Crohn's disease in US women. Ann Rheum Dis 72(7):1200-1205

30. Cohen AD, Dreiher J, Birkenfeld S (2009) Psoriasis associated with ulcerative colitis and Crohn's disease. J Eur Acad Dermatol Venereol 23(5):561-565

31. Tsai TF, Wang TS, Hung ST, Tsai PI, Schenkel B, Zhang M, Tang CH (2011) Epidemiology and comorbidities of psoriasis patients in a national database in Taiwan. J Dermatol Sci 63(1):40-46

32. McCarthy FP (1949) Pyostomatitis vegetans; report of three cases. Arch Derm Syphilol 60(5, Pt. 1):750-764

33. Femiano F, Lanza A, Buonaiuto C, Perillo L, Dell'Ermo A, Cirillo N (2009) Pyostomatitis vegetans: A review of the literature. Med Oral Patol Oral Cir Bucal 14(3):E114-E117

34. Truchuelo MT, Alcántara J, Vano-Galván $S$, Jaén P, Moreno C (2013) Bowel-associated dermatosis-arthritis syndrome:another cutaneous manifestation of inflammatory intestinal disease. Int JDermatol 52(12):1596-1598

35. Defilippis EM, Magro C, Jorizzo JL (2014) Bowelassociated dermatosis-arthritis syndrome in a patient with ulcerative colitis: an extraintestinal manifestation of inflammatory bowel disease. Clin J Gastroenterol 7(5):410-413

36. Monshi B, Stockinger T, Vigl K, Richter L, Weihsengruber F, Rappersberger K (2015) Phrynoderma and acquirded acrodermatitis enteropathica in breastfeeding women after bariatric surgery. JDtsch Dermatol Ges 13(11):1147-1154

37. von Felbert $V$, Hunziker T (2010) Acrodermatitis enteropathica-like skin lesions due to Crohn's disease-associated zinc deficiency. Hautarzt 61(11):927-929
Auch online Zugang zu allen Beiträgen Ihres Individualabonnements

Wussten Sie, dass Sie als Abonnent dieser Zeitschrift automatisch online Zugriffsrechte auf das gesamte Beitragsarchiv und die CME-Kurse haben?

Der Zugang zu Ihrer Online-Bibliothek und den CME-Kursen ist im Abonnement Ihrer Zeitschrift inbegriffen. Neben den gedruckten Ausgaben, können Sie Themen einfach am Computer recherchieren. Für den Zugang müssen Sie sich lediglich einmal über www.springermedizin.de/register registrieren.

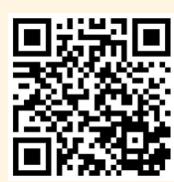

Über diesen QR-Code schnell und einfach registrieren

Für die Registrierung brauchen Sie nur

- die Nummer Ihres Abonnements

(siehe Adressaufkleber auf Ihrem Heft) und - Ihre Lieferanschrift.

So stellen Sie die Zugehörigkeit zu Ihrer Zeitschrift sicher.

Nach Login erhalten Sie dann sofort die Online-Rechte für den Volltextzugriff und den Zugang zu Ihren CME-Kursen.

Mit Benutzername und Passwort haben Sie außerdem Zugang zu den freien Inhalten auf den Seiten von: https://www.springermedizin.de/ http://www.aerztezeitung.de/ http://www.heilberufe-online.de/

Sollten Fragen oder Probleme auftauchen, wenden Sie sich einfach an Ihren Kundenservice über:

kundenservice@springermedizin.de 